UCRL-JC-128897

PREPRINT

\title{
Laboratory Measurements of Resonant Contributions to Fe XXIV Line Emission
}

\author{
M. F. Gu, P. Beiersdorfer, G. V. Brown, S. M. Kahn, \\ D. A. Liedahl, K. J. Reed, D. W. Savin
}

This paper was prepared for submittal to the International Conference on Atomic Data and Molecular Data Gaithesburg, MD

September 29-October 2, 1997

October 27, 1997

This is a preprint of a paper intended for publication in a journal or proceedings. Since changes may be made before publication, this preprint is made available with the understanding that it will not be cited or reproduced without the permission of the author. 


\section{DISCLAIMER}

This document was prepared as an account of work sponsored by an agency of the United States Government. Neither the United States Government nor the University of California nor any of their employees, makes any warranty, express or implied, or assumes any legal liability or responsibility for the accuracy, completeness, or usefulness of any information, apparatus, product, or process disclosed, or represents that its use would not infringe privately owned rights. Reference herein to any specific commercial product, process, or service by trade name, trademark, manufacturer, or otherwise, does not necessarily constitute or imply its endorsement, recommendation, or favoring by the United States Government or the University of California. The views and opinions of authors expressed herein do not necessarily state or reflect those of the United States Government or the University of California, and shall not be used for advertising or product endorsement purposes. 


\title{
Laboratory Measurements of Resonant Contributions to Fe XXIV Line Emission
}

\author{
M.F. Gu ${ }^{1}$, P. Beiersdorfer ${ }^{2}$, G.V. Brown ${ }^{2}$, S.M. Kahn ${ }^{1}$, \\ D.A. Liedahl' ${ }^{2}$, K.J. Reed ${ }^{2}$ and D.W. Savin ${ }^{1}$ \\ ${ }^{1}$ Columbia Astrophysics Laboratory, Columbia University, New York, NY 10027 \\ ${ }^{2}$ Lawrence Livermore National Laboratory, Livermore, CA 94550
}

\section{INTRODUCTION}

Recent Advanced Satellite For Cosmology and Astrophysics (ASCA) observations of the Centaurus cluster of galaxies [1] exhibit discrepancies with the relative line intensities of various Fe XXIII and XXIV $L$-shell emission lines predicted by standard plasma codes. This discrepancy is seen with all standard emission codes and is attributed to problems with the atomic physics used in the emission codes. New distorted wave (DW) calculations of Fe XXIII and Fe XXIV electron impact excitation (EIE) rate coefficients [2] appear to provide better agreement with the $A S C A$ observations. Using the Lawrence Livermore electron beam ion trap (EBIT), Savin et al. [3] measured the relative line emission of several Fe XXIV $3 \rightarrow 2$ and $4 \rightarrow 2$ lines and found good agreement with the calculations of Liedahl et al. These measurements, however, were carried out at electron energies significantly greater than the excitation threshold energies of the lines observed. The calculations remain to be experimentally verified for energies at which resonant processes may be important and for energies near threshold where DW calculations may overpredict the EIE cross section.

A number of X-ray astronomy satellites are scheduled for launch in the next few years. The Advanced X-ray Astrophysics Facility $(A X A F)$ is scheduled for launch in 1998, and the X-Ray Multi-mirror Mission (XMM) and Astro-E in 1999. These satellites will carry spectrometers with resolving powers in the $\mathrm{Fe} L$-shell emission region over an order of magnitude greater than the spectrometers aboard $A S C A$. Interpreting $A X A F, X M M, A s t r o-E$ spectra will require atomic data at an accuracy significantly greater than the data presently used in the standard emission codes.

To address some of the existing and upcoming needs of X-ray astrophysics, we have continued our studies of Fe XXIV line emission. In this work, we measured Fe XXIV $3 \rightarrow 2$ line emission at energies around threshold, using EBIT to examine the resonance contributions to the line emissivity. Here we present relative cross sections, at electron energies between 700 and $1500 \mathrm{eV}$, for producing line emission at wavelength $\lambda=11.18 \AA$ of the Fe XXIV $3 d_{5 / 2} \rightarrow 2 p_{3 / 2}$ transition.

Various processes can contribute to line emission observed from a collisional plasma. Direct excitation (DE) is the most important one at energies above the EIE threshold. Below threshold, Dielectronic recombination (DR) produces high $n$ satellites which cannot be resolved from the EIE line. Resonant excitation (RE) can populate the same levels as DE via dielectronic capture followed by autoionization to the level of interest. In this work, we have measured the following processes:

- DE

$$
F e^{23+}(2 s)+e^{-} \rightarrow F e^{23+}\left(3 d_{5 / 2}\right)+e^{-} \rightarrow F e^{23+}\left(2 p_{3 / 2}\right)+h \nu_{1}+e^{-}
$$


Table 1: Uncertainties for observed line emission measurements.

\begin{tabular}{|r|c|}
\hline Source & $\begin{array}{c}\lambda=11.18 \AA \\
\text { (percent) }\end{array}$ \\
\hline Statistics & $8-14$ \\
Background Subtraction & 5 \\
Polarization Effects & 5 \\
Electron Density & 2 \\
Normalization & 10 \\
\hline Quadrature Sum & $15-19$ \\
\hline
\end{tabular}

- $\mathrm{RE}$ $F e^{23+}(2 s)+e^{-} \rightarrow F e^{22+}\left(4 l^{\prime} n l^{\prime \prime}\right) \rightarrow F e^{23+}\left(3 d_{5 / 2}\right)+e^{-} \rightarrow F e^{23+}\left(2 p_{3 / 2}\right)+h \nu_{1}+e^{-}$

- DR

$$
F e^{23+}(2 s)+e^{-} \rightarrow F e^{22+}\left(3 d_{5 / 2} n l^{\prime}\right) \rightarrow F e^{22+}\left(2 p_{3 / 2} n l^{\prime}\right)+h \nu_{2}+e^{-}
$$

Quantum mechanically, there is no way to distinguish between DE and RE, and we measure the coherent sum of the two processes.

\section{EXPERIMENTAL TECHNIQUE}

EBIT [4] uses a magnetically confined electron beam to produce a potential well which traps the ions in the radial direction. In the axial direction, ions are trapped by voltages applied to the top and bottom drift tubes. The electron beam is also used to ionize and excite the trapped ions. Radiative decay of the excited ions results in X-ray emission which is observed by a flat crystal spectrometer (FCS) through one of the X-ray ports. A thallium hydrogen phthalate (001) crystal is used. The dispersion plane of the FCS is perpendicular to the electron beam. Between EBIT and the FCS is a $4 \mu \mathrm{m}$ polypropylene window. X-rays are detected by a flowing gas $(90 \% \mathrm{Ar}$ and $10 \%$ $\mathrm{CH}_{4}$ at 1 atmosphere) position sensitive proportional counter with a $4 \mu \mathrm{m}$ polypropylene window.

After injecting iron into EBIT, the electron beam energy is kept at $4 \mathrm{keV}$ for $200 \mathrm{~ms}$. Then every $10 \mathrm{~ms}$ the beam energy is swept between 700 and $1500 \mathrm{eV}$ using a sawtooth pattern $2 \mathrm{~ms}$ in length. The intensity of a particular line is $I \propto \int d \mathbf{r} n_{e}(\mathbf{r}) n_{q}(\mathbf{r}) \sigma v$, where $n_{e}(\mathbf{r})$ is the electron density, $n_{q}(\mathbf{r})$ is the ion density, $\sigma$ is the cross section for producing the observed line emission and $v$ is the electron velocity. During the sweeping of the beam energy, the charge balance changes insignificantly because the ionization and recombination time scales at these energies are $\geq 100 \mathrm{~ms}$. We maintain a nearly constant $n_{e}(\mathbf{r})$ by varying the anode voltage of electron gun. We record the intensity of line emission at $\lambda=11.18 \AA$ as a function of beam energy. Because $\int d \mathbf{r} n_{e}(\mathbf{r}) n_{q}(\mathbf{r})$ is kept constant versus $E$, we can derive cross sections for producing these lines by normalizing our measurements to theory.

\section{UNCERTAINTY ANALYSIS}

Uncertainties are listed in Table 1 at $1 \sigma$ confidence level.

There are about 200 counts per beam energy bin $(\sim 12 \mathrm{eV}$ per bin) for the line emission at $\lambda=11.18 \AA$ above the DE threshold. The DR satellites have peak counts of between 50 and 100 . The $1 \sigma$ statistical uncertainty varies between $8 \%$ and $14 \%$. 


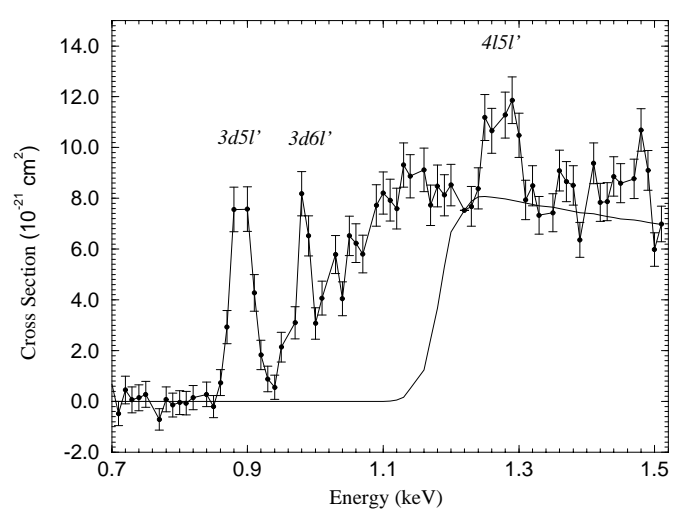

Figure 1: Normalized cross sections for producing line emission at $\lambda=11.18 \AA$.

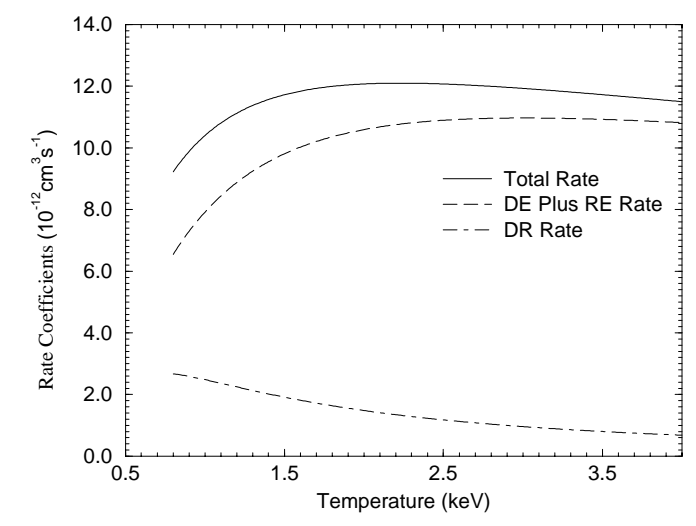

Figure 2: Rate coefficients for producing line emission at $\lambda=11.18 \AA$.

We observed line emission due to charge transfer (CT) off of neutal gas in EBIT. This signal is independent of beam energy. At beam energies between 700 and $800 \mathrm{eV}$, the CT signal is clearly resolved from DR resonances and seen to be constant. We subtract this background from the total intensity. The relative uncertainty introduced by this subtraction is about $5 \%$.

Line emission produced by a unidirectional electron beam is linearly polarized and anisotropic [5]. Crystals have different integrated reflectivities for X-rays polarized parallel to and perpendicular to the dispersion plane [6]. The observed line intensities depend on the polarization of the emitted lines. This is a function of collision energy. In the energy range covered here, polarization due to DE does not change significantly. Polarization of the DR satellites should approach that of DE as the beam energy approaches the DE threshold [7]. RE is expected to be less polarized than DE emission. We assume the line emission at all energies has the same polarization. Polarization effects need to be further investigated but are estimated to introduce $<5 \%$ uncertainty.

While sweeping the electron beam energy, we try to keep the electron density constant by ramping the extraction voltage of the electron gun in sync with the beam energy. The measured beam current indicates that the electron density varies by less than $10 \%$ between 700 and 1500 $\mathrm{eV}$. We have corrected the observed line intensities for this variation. The electrons in EBIT have a transverse energy of $\sim 150 \mathrm{eV}[8]$. The resulting change of the pitch angle between the electron velocity and the beam direction results in variation of electron density versus beam energy. Using the normalization energy as our reference point, $n_{e}$ varies by $\pm 2 \%$ across the measured energy range due to the changing pitch angle.

Our normalization point lies $\sim 40 \mathrm{eV}$ above the $\mathrm{DE}$ threshold and $\geq 40 \mathrm{eV}$ away from all $\mathrm{RE}$ resonances. Given the electron beam energy spread of $50 \mathrm{eV}[9]$, we estimate that emission at the normalization point is not significantly affected by either DR just below the DE threshold or the RE resonances. Thus we assume the line emission is completely due to DE at this energy and use HULLAC [2] calculated cross sections. Statistical uncertainties at the normalization energy introduce uncertainties of $\sim 10 \%$ in the cross sections for line emission at $\lambda=11.18 \AA$.

\section{RESULTS}

Figure 1 shows the normalized cross section for producing $\lambda=11.18 \AA$ line emission. The error bars on the data points show the $1 \sigma$ statistical uncertainties. The normalization point is presented without error bar. The $3 l 5 l^{\prime}$ and $3 l 6 l^{\prime} \mathrm{DR}$ resonances are well separated. All other DR resonances between $1.0 \mathrm{keV}$ and the $\mathrm{DE}$ threshold are unresolved and appear as a continuous increase in the 
cross sections. The RE resonance at a beam energy of about $1.25 \mathrm{keV}$ is produced by dielectronic capture into the Fe XXIV $4 l 5 l^{\prime}$ level followed by autoionizing to the $3 d_{5 / 2}$ level.

Using the relative cross sections obtained above, we calculate the rate coefficients of each process. The DE rate is calculated by using HULLAC [2] cross sections. For experimentally inferred rates, only line emission within the measured energy range is included. Figure 2 shows the experimentally inferred rates for DR, DE plus RE, and the total rates. The total rate includes the experimental inferred DR, DE plus RE, and the theoretical DE rates at energies outside of our measured range. The theoretical DE rates do not include the cascade contributions. At a temperature of $1.7 \mathrm{keV}$, near where Fe XXIV emissivity peaks, DR contributions amount to $\sim 20 \%$ for $\lambda=11.18 \AA$ line emission. RE enhances the rates by $\sim 5 \%$. However, the unmeasured $\mathrm{RE}$ contributions at higher energies can be expected to increase the rates further. Thus, plasma emission codes which do not include the effect of DR and RE on line emissivities can be expected to underestimate line intensity by at least $\sim 25 \%$.

\section{ACKNOWLEDGMENTS}

The authors wish to thank S. T. Manson for stimulating conversations and P. A. D'Antonio, E. W. Magee, and D. H. Nelson for their expert technical support. Work at Lawrence Livermore National Laboratory was performed under the auspices of the US Department of Energy (contract W-7405ENG-48). This program is supported by a NASA High Energy Astrophysics X-Ray Astronomy Research and Analysis grant NAGW-4185 (Columbia University) and work order W-19127 (LLNL).

\section{References}

[1] Fabian, A. C., et al. 1994, ApJ, 436, L63

[2] Liedahl, D.A., et al. 1995, ApJ, 438, L115

[3] Savin, D.W., et al. 1996, ApJ, 470, L73

[4] Levine, M. A., et al. 1988, Phys. Scr., T22, 157

[5] Percival, I. C., \& Seaton, M. J. 1958, Philos. Trans. R. Soc. London, A, 251, 113

[6] Henke, B. L., Gullikson, E. M., \& Davis, J. C. 1993, At. Data Nucl. Data Tables, 54, 181

[7] Inal, M. K. and Dubau, J. 1989, J. Phys B. 22, 3329

[8] Marrs, R. E. (Private Communication)

[9] Beiersdorfer, P., et al. 1992, Phys. Rev. A46, 3812 


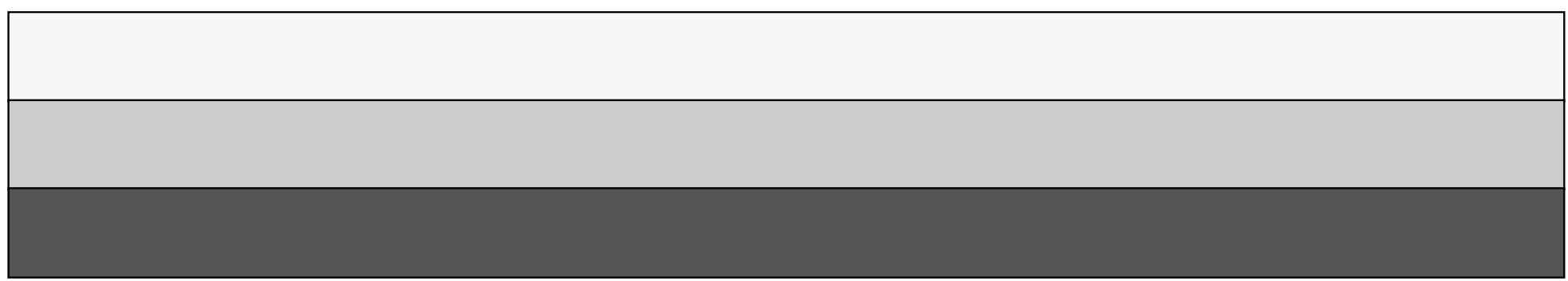

